\title{
Randomized Controlled Trial on the Use of Intravenous Immune Globulin in Acute Pediatric Myocarditis
}

\section{Sonia Ali El-Saiedi*}

Cairo University, Cairo, Egypt

\begin{abstract}
Background: Myocardial damage in myocarditis is mediated, in part, by immunological mechanisms. This prospective placebo controlled trial was designed to determine whether Intravenous Immune Globulin (IVIG) improves Left Ventricular Fractional Shortening (LVFS) and decrease left ventricular end diastolic volume (LVED) in children with acute onset dilated cardiomyopathy or myocarditis.

Methods: 86 patients were enrolled in the study all presenting with recent onset dilated cardiomyopathy duration less than 6 months. They were randomized to $2 \mathrm{~g} / \mathrm{kg} \mathrm{IVIG} \mathrm{over} \mathrm{two} \mathrm{consecutive} \mathrm{days} \mathrm{or} \mathrm{placebo.} \mathrm{All} \mathrm{underwent}$ echocardiographic examination before admission then one month and six months after randomization. Each time the left ventricular end diastolic dimension (LVEDD) and fractional shortening (FS) were recorded.

Results: At baseline the LVEDD and FS did not differ significantly between those treated with IVIG and those treated with Placebo. Over the period of six months follow-up the patients in both groups became better. Patients treated with conventional therapy continued to have a mean end diastolic dimensions significantly larger than treated children, with no evidence of regression.

Conclusion: The results suggest that for young patients with recent onset dilated cardiomyopathy, IVIG does not augment the improvement in left ventricular FS. However, in this overall cohort, left ventricular FS improved significantly with time during follow up.
\end{abstract}

Keywords: Randomized controlled trial; Intravenous immunoglobulin; Myocarditis; Cardiomyopathy; Pediatric acute myocarditis

\section{Introduction}

The natural history of acute myocarditis is largely unknown. Data about its short and long term evolution, especially in children, are limited. Acute myocarditis is rarely symptomatic; it is difficult to identify subclinical episodes and their possible evolution to Dilated Cardiomyopathy (DCM). The clinical manifestations remain quite indistinguishable from idiopathic dilated cardiomyopathy [1].

Myocardial damage in myocarditis is mediated, in part, by immunological mechanisms. Secondary autoimmune pathogenesis involving both cellular and humoral pathways might have a greater role in altering myocardial function than the viral infection itself [2]. Initial efforts to limit the development of cardiac dilatation and dysfunction in patients with inflammatory myocarditis by immunosuppressive therapy were successful [3].

High dose intravenous immune globulin (IVIG) has both antiviral and immune modulatory effects and is an important therapy for Kawasaki Disease [4]. Its utility for this disorder led to its use in children with new onset dilated cardiomyopathy and myocarditis. Drucker et al. [5] reported a series of children treated with immune globulin in which significant improvements in left ventricular function were seen compared with recent-onset dilated cardiomyopathy. On the other hand, a randomized controlled trial designed to determine whether IVIG improves left ventricular ejection fraction in adults with recent onset dilated cardiomyopathy [6] did not demonstrate evidence of therapeutic efficacy for immune globulin administration in this group of patients.

In the cardiomyopathy clinic of Cairo University Children Hospital (tertiary care centre) we are faced with a large number of cases per year $(>60)$ presenting with recent onset dilated cardiomyopathy. The limited resources the hospital has made it difficult to provide IVIG for all such patients. This is complicated by the fact that the use of IVIG is controversial in the medical literature. Accordingly, a prospective randomized study was performed to evaluate the potential role of intravenous immune globulin therapy in childhood acute onset dilated cardiomyopathy and to determine the best timing for its usage.

\section{Patients and Methods}

The study was a prospective randomized placebo-controlled evaluation of the addition of IVIG to conventional therapy (inotropes, digoxine, diuretics, after load reduction drugs) in young patients with acute onset dilated cardiomyopathy presenting to Cairo university children hospital, cardiomyopathy clinic over five-year period. The inclusion criteria were: (1) patients less than 15 years old, (2) FS less than $20 \%$, (3) duration of cardiac symptoms not more than 6 months at the initial time of randomization. Patients presenting in the neonatal period or giving history of cardiac symptoms since birth were excluded. All patients were hospitalized in the inpatient wards. Treatment assignment was performed by a third party using a random table block (initial block of 2-treatment) stratified by clinical centre. All patients signed the consent to join the study. Those who did not sign the consent were excluded from the study. The Study was approved by the ethics committee of Cairo University.

*Corresponding author: Sonia Ali El-Saiedi, Cairo University, Cairo, Egypt, E-mail: myheartclinic@windowslive.com

Received October 17, 2013; Accepted November 28, 2013; Published December 07, 2013

Citation: El-Saiedi SA (2013) Randomized Controlled Trial on the Use of Intravenous Immune Globulin in Acute Pediatric Myocarditis. J Clin Res Bioeth 5: 170. doi:10.4172/2155-9627.1000170

Copyright: (c) 2013 El-Saiedi SA. This is an open-access article distributed under the terms of the Creative Commons Attribution License, which permits unrestricted use, distribution, and reproduction in any medium, provided the original author and source are credited. 
Patients randomized to the treatment group (42 patients) received a total of $2 \mathrm{~g} / \mathrm{kg}$ IVIG (VIGAM-S. BPL, $2.5 \mathrm{~g}$ and $5 \mathrm{~g}$ ) [7]. This was administered at $1 \mathrm{~g} / \mathrm{kg}$ IV each day for two consecutive days. Patients randomized to placebo (44 patients) received regular glucose $5 \%$ IV fluids $10 \mathrm{ml} / \mathrm{kg}$ repeated on two consecutive days.

Patients included were seen during follow up at 1 and 6 months after randomization.

\section{Statistical Analysis}

The primary end point was determined by change in FS and LVEDD from baseline to 6 months after randomization. Secondary end point was survival versus death.

The study planned sample size of 40 patients or more in each treatment arm calculated to provide $95 \%$ power to detect a treatment difference in FS change score of $>5 \%$ using a Two-sided test with significance level of 0.05 . These projected differences and variability estimates were based on the results reported from two previous studies $[5,6]$.

Statistical package of social science (SPSS) version 9 was used for analysis of data. Data summarized as mean, SE. Chi-square was used for analysis of quantitative data. Non-parametric test (Mann- Whitney) was used for analysis of 2 independent quantitative variables and two ways ANOVA (Analysis of variance) for comparison of 2 groups over time. Followed by post hoc test to detect difference over time in each group separately. P-value was considered significant if $\leq 0.05$.

\section{Results}

All patients included had an age range from 4 months to 6 years and a mean of $2.08 \pm 1.28$ yrs in the IVIG group vs. $3.29 \pm 1.9$ in the placebo group. There was no significant difference between the two groups regarding male and female distribution. The duration of illness before randomization was constantly less than or equal to 6 months with a mean of $3.1 \pm 1.6$ months for patients who received IVIG and $4.02 \pm 1.5$ months in patients who received placebo with no significant difference between the two groups.

Table 1 show the echocardiographic, ECG and laboratory data of patients in both groups. Patients who received IVIG and those who did not were comparable at base-line in all clinical parameters. Cardiac enzymes were high in both groups with a higher level in the patients who did not receive IVIG. None of the patients included presented mainly with arrhythmia or was post cardiac arrest.

Patients were classified using the New York Heart Association

\begin{tabular}{|c|c|c|c|}
\hline Variables & IVIG (mean \pm SE) N=42 & Placebo (mean \pm SE) N=44 & P value \\
\hline FS\% & $16.6 \pm 0.6$ & $17.3 \pm 0.8$ & $>0.05$ \\
\hline LVEDD $(\mathrm{mm})$ & $44.7 \pm 1.2$ & $47.5 \pm 1.6$ & $>0.05$ \\
\hline $\mathrm{Hb}(\mathrm{gm} / \mathrm{dl})$ & $10.3 \pm 0.2$ & $10.2 \pm 0.2$ & $>0.05$ \\
\hline $\mathrm{WBCs}$ & $10.1 \pm 0.6$ & $9.2 \pm 0.6$ & $>0.05$ \\
\hline $\mathrm{ESR}$ & $16.1 \pm 1.7$ & $14.8 \pm 1.3$ & $>0.05$ \\
\hline $\mathrm{CPK}(\mathrm{U} / \mathrm{L})$ & $155.6 \pm 33.9$ & $243.2 \pm 34.2$ & $0.002^{*}$ \\
\hline $\mathrm{LDL}(\mathrm{U} / \mathrm{L})$ & $534.0 \pm 83.9$ & $644.8 \pm 174.7$ & $>0.05$ \\
\hline $\mathrm{CKMB}(\mathrm{U} / \mathrm{L})$ & $91.2 \pm 33.6$ & $130.8 \pm 76.2$ & $>0.05$ \\
\hline $\mathrm{HR}$ & $123.4 \pm 3.9$ & $126.4 \pm 3.2$ & $>0.05$ \\
\hline P-R interval & $143.6 \pm 11.6$ & $127.3 \pm 2.7$ & $>0.05$ \\
\hline QRS duration & $85.4 \pm 1.7$ & $96.6 \pm 5.8$ & $>0.05$ \\
\hline
\end{tabular}

Table 1: Comparison between ECHO parameters, laboratory data and ECG findings in patients randomized to IVIG and placebo.

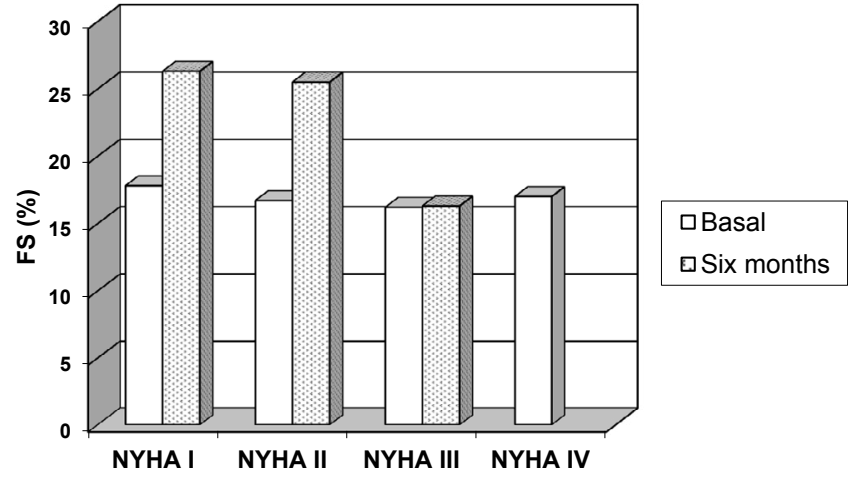

Figure 1: FS in relation to NYHA class in all patients ( IVIG and Placebo) presenting with heart failure.

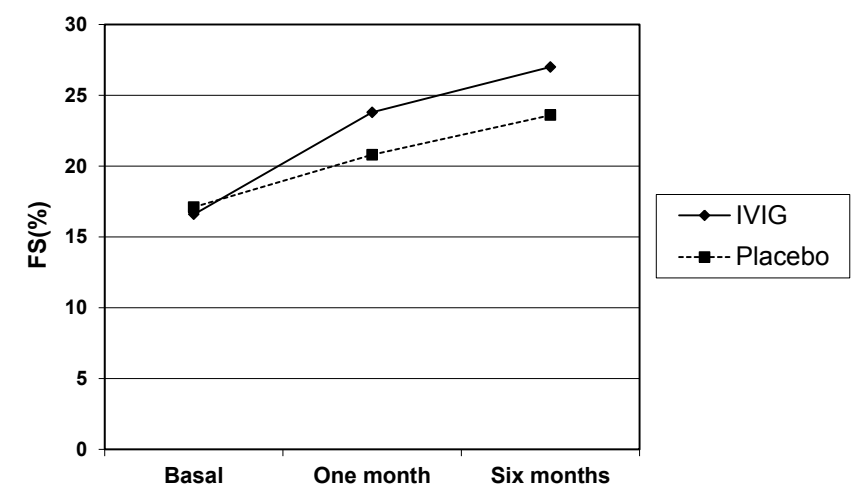

Figure 2: Mean value of FS of patients with dilated cardiomyopathy receiving IVIG vs Placebo.

classification of heart failure (NYHA).There were 69 patients $(80.2 \%)$ NYHA class II ( 35 IVIG vs. 34 placebo), 10 cases NYHA class I (4 IVIG vs. 6 Placebo) only 5 patients were in NYHA class III (3 IVIG vs. 2 Placebo), while two patients were NYHA class IV both in the placebo group. Using NYHA class as a predictor of outcome, it was evident that patients presenting in either NYHA class I or II showed higher FS values after 6 months of treatment than those presenting in NYHA class III while those presenting in Class IV all died (Figure 1).

All our patients presented with severely impaired left ventricular function as reflected by FS less than $20 \%$ and dilated left ventricle (increased LVEDD).

We explored the effect of IVIG treatment on LVEDD and on FS. At baseline the LVEDD and FS (Table 1) did not differ significantly between those treated with IVIG and those treated with placebo. Over a period of six months, patients in both groups improved (Figures 2 and 3). Twoway ANOVA of effect of time on FS was highly significant in the group who received IVIG $(\mathrm{P}<0.0001)$. Less significant improvement was noted with time in patients who received placebo. LVEDD improved with time to a significant degree in the IVIG group $(\mathrm{P}=0.001)$. The improvement in both parameters did not show any significant difference between both groups when studying the time effect versus group. Patients treated with placebo continued to have mean end diastolic dimensions significantly larger than treated children with IVIG ( $48.1 \pm 2.1$ vs. 41.3 \pm 2.4 ) with no evidence of regression (Figure 3).

To answer the question of the best timing for adding IVIG we 


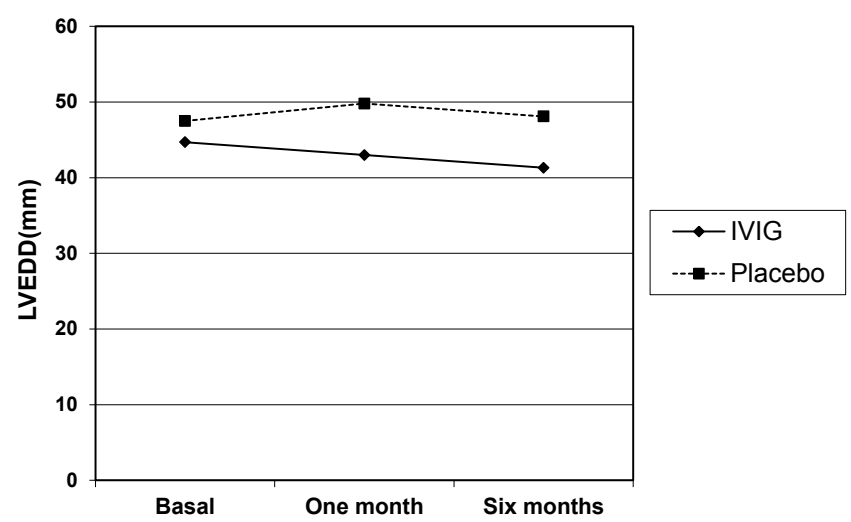

Figure 3: Mean value of LVEDD of patients with dilated cardiomyopathy receiving IVIG vs Placebo.

\begin{tabular}{|c|c|c|c|}
\hline Group & One month & Six months & P-value \\
\hline Patients IVIG & $85.3 \%$ & $85.3 \%$ & \multirow{2}{*}{0.3} \\
\hline Patients Placebo & $81.8 \%$ & $70.1 \%$ & \\
\hline
\end{tabular}

Table 2: Survival rate.

\section{Survival Functions}

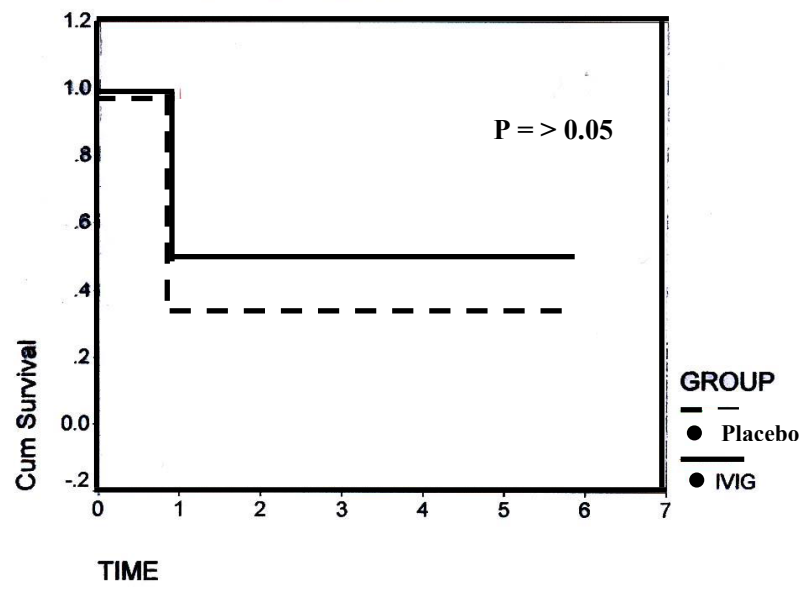

Figure 4: Kaplan-Meier estimates of survival among patients treated with IVIG vs placebo. Each step represents death.

divided patients who received IVIG into two groups; those who received the IVIG within the first 2 months of illness and those who received it after two months. It is worth noting that the patients who received IVIG after the first two months had a significant improvement in FS compared to those who received the treatment early on.

Although survival tended to be better among IVIG treated patients, the difference in overall survival did not achieve statistical significance in our sample (Table 2). Figure 4 shows the Kaplan-Meier survival curve demonstrating that most deaths in both groups occurred during the first weeks after diagnosis whether IVIG was given or not. No major adverse effects of IVIG administration was noted in any of our patients. We could not detect worsening of congestive heart failure as anticongestive therapy had already been instituted in all patients. Neither hypotension nor anaphylaxis was noted in any patient. Few patients had flu-like symptoms.

\section{Discussion}

In recent years $\mathrm{CUCH}$ has been receiving ever-increasing numbers of myocarditis or acute onset dilated cardiomyopathy. According to the 'hygiene hypothesis', the de-creasing incidence of infections in western countries and more recently in developing countries like Egypt, is at the origin of the increasing incidence of both autoimmune and allergic diseases [8]. It was found that it was possible to extend the hypothesis from the field of allergy, where it was formulated, to that of autoimmune diseases such as type 1 diabetes (T1D) or Multiple Sclerosis (MS) [9]. On the other hand, IVIG is a relatively expensive drug; accordingly doctors working with limited resources have to insure the cost-benefit of the addition of such therapy to all such patients.

The possible therapeutic role of immune modulation is consistent with studies in animal models. The autoimmune pathogenesis of virally initiated inflammatory heart disease has been extensively studied in a murine model of coxackie virus induced myocarditis [10]. High dose immune globulin markedly improves survival when given at the time of viral infection, even though it does not reduce viral titres [11]. Since 1990, the routine management of presumed acute myocarditis at Children's Hospital, Boston, and Children's Hospital, Los Angeles, has included high-dose IVIG [5], followed by other pediatric hospitals elsewhere in the world.

The mechanism by which IVIG may improve myocardial dysfunction in myocarditis is unknown. IVIG could be effective by providing specific antibodies to viruses and thus could lead to more rapid clearing of myocardial viral infection [11]. An alternative mechanism of gamma globulin action may be modulation of the immune re-sponse leading to decreased cardiac inflammation or to down regulation of pro-inflammatory cytokines that have direct negative inotropic effects [12-14]. It appears that the negative inotropic effects results from the generation of nitric oxide induced by various cytokines. IVIG induces synthesis of the interleukin-1 receptor antagonist, and this may result in the down regulation of nitric oxide synthetase [12]. Previous reports have confirmed that immunoadsorption and subsequent IgG substitution improves cardiovascular function in DCM [15].

The use of other immunosuppressive agents for treatment of myocarditis is controversial; both beneficial and deleterious effects have been noted [16,17]. Parillo et al. [18] found an initial improvement in ventricular function in patients with dilated cardiomyopathy who received steroids compared with control subjects who received conventional therapy; however, there was no significant difference in ventricular function between the two groups after lapse of six months from treatment. It has been demonstrated in animal models that if steroids were used during the viral stage myocardial damage is enhanced [19]. Recently, after the initiation of this study Al-Saeed and Dilawar [20] suggested that use of high dose IVIG in combination with corticosteroid $(10 \mathrm{mg} / \mathrm{Kg} /$ day methyl prednisone followed by Prednisone $2 \mathrm{mg} / \mathrm{kg} /$ day then weaned gradually) for the treatment of acute myocarditis is an effective treatment for acute viral myocarditis in pediatric population.

In this prospective randomized controlled study of IVIG treatment of presumed acute myocarditis in children, treatment with IVIG was not associated with a statistically significant difference in both left ventricular function indices (LVEDD and FS). IVIG administration was also associated with a trend towards improvement of survival, which was not significantly different from other patients who received only conventional therapy. All myocarditis patients improved significantly with time on continuing medical therapy. 
In past reports, spontaneous improvement has been described in up to $70 \%$ of suspected cases of endemic viral myocarditis diagnosed entirely on clinical grounds [21]. After the introduction of endomyocardial biopsy, the real incidence remains uncertain. Dec et al. [22], as well as Olsen et al. [23], reported spontaneous improvement in $40-50 \%$ of patients with histologically confirmed acute myocarditis. In another experience of 54 patients with biopsy- proven acute myocarditis, the overall incidence of spontaneous improvement was $50 \%$, and improvement was more common among patients with chest pain or arrythmias at the onset [24]. However, studies based on clinical diagnosis alone should be differentiated from those based on endomyocardial biopsy findings, as well as from studies investigating patients treated with or without immunosuppressive drugs [25].

Myocarditis treatment overview published recently concluded that patients with sub-acute myocarditis and new onset dilated cardiomyopathy and HF often improve spontaneously with standard HF management. The investigators recommended endomyocardial biopsy to all cases especially those resistant to regular treatment [26]. Thus the frequent spontaneous improvement in cases of acute myocarditis should be borne in mind when deciding whether to start antiviral treatment or immune modulation therapies.

In the two studies done by McNamara in patients with new onset dilated cardiomyopathy, the patients who received IVIG did not show significant LVEF improvement. However, in his overall series, LVEF improved significantly during follow up with or without IVIG addition $[6,27]$.

Using NYHA class, as a predictor of outcome by baseline parameter in the 86 patients included in this study, data revealed that patients in class I and II had good recovery with marked increase in FS and slight decrease in LVEDD. Patients in class III showed a slight increase in FS and worsening of the LVEDD while all patients in class IV died within six months. This shows that the NYHA class is a good predictor of outcome. McNamara et al. in 2001 have shown similar findings with fewer survivors from patients in class III or IV [6].

It is known that some non-invasive variables evaluated at clinical presentation show good predictive accuracy in this subset of patients with acute myocarditis. These include ECG alterations, such as QRS changes, atrial fibrillation, or low voltages that may predict an unfavourable clinical course of acute myocarditis, reflecting more extensive myocardial injury $[28,29]$. We studied patients in the two groups regarding the ECG parameters, QRS duration and PR interval, that proved not to be a sensitive predictor of outcome. Similarly the level of increase in cardiac enzymes was not a sensitive variable.

Myocarditis is a triphasic disease process: phase 1, the viral stage, phase 2, the auto-immunity and phase 3 the dilated cardiomyopathy phase. It is expected that IVIG in myocarditis would be effective in either the viral stage or the autoimmunity stage be-fore progression to phase three [2].

The present work tackled the problem of timing of treatment; it was worth noting that the patients who received IVIG after the first two months had a significant improvement in FS compared to those that received the drug early on. This can be explained by its better effect on resistant types of myocarditis stressing the importance of endomyocardial biopsy.

It is well known that myocarditis patients may recover, develop DCM, or die [30].

In this series, twelve patients (27.5\%) died from the patients who had only the regular treatment measures plus placebo while six patients (14\%) from the group that was put early on IVIG. It seems that survival was better in those who received IVIG but not reaching a statistically significant level. It is worth noting that most deaths occurred within the first 4-5 weeks after diagnosis and starting management whether IVIG was given or not.

In a recently published paper the authors identified knowledge gaps and opportunities in the treatment of myocarditis. They concluded that a serious research should: (1) determine when and how to discontinue standard heart failure therapy in patients with recovering LV functions. (2) Identify patient cohorts who will benefit from tailored an-tiviral or immunosuppressive therapy. (3) Provide an optimal time frame for initiatia-tion of immunosuppressive treatment [30].

It is evident that this work covers some of these knowledge gaps.

\section{Conclusion}

The present study did not demonstrate evidence of therapeutic efficiency for immune globulin administration for acute onset myocarditis in children. However, treatment with standard therapies resulted in improvement with time in all patients.

At present, the optimal time frame for starting immune modulation therapy is speculative because of lack of data. This work pointed that IVIG given after the first two months gives a significant improvement in FS.

Efforts should still be directed towards defining the stages of myocarditis and finding novel diagnostic strategies that more fully reflect the underlying pathophysiologic stage. New advanced methods to characterize active autoimmunity, with high sensitivity and specificity, are needed to be able to classify our patients and pick more accurately those who might benefit mostly from immune modulation.

\section{Acknowledgements}

To Dr. Ibrahim Fayez consultant epidemiologist, WHO; and Professor Dr. Enas El Attar member of statistics department Cancer Institute, Cairo. They were responsible for planning the study, insuring proper randomization and finally doing the statistical analysis.

\section{References}

1. Kearney MT, Cotton JM, Richardson PJ, Shah AM (2001) Viral myocarditis and dilated cardiomyopathy: mechanisms, manifestations, and management. Postgrad Med J 77: 4-10.

2. Liu PP, Mason JW (2001) Advances in the understanding of myocarditis Circulation 104: 1076-1082.

3. Mason JW, O'Connell JB, Herskowitz A, Rose NR, McManus BM, et al. (1995) A clinical trial of immunosuppressive therapy for myocarditis. The Myocarditis Treatment Trial Investigators. N Engl J Med 333: 269-275.

4. Newburger JW, Takahashi M, Burns JC, Beiser AS, Chung KJ, et al. (1986) The treatment of Kawasaki syndrome with intravenous gamma globulin. $\mathrm{N}$ Engl $\mathrm{J}$ Med 315: 341-347

5. Drucker NA, Colan SD, Lewis AB, Beiser AS, Wessel DL, et al. (1994) Gammaglobulin treatment of acute myocarditis in the pediatric population. Circulation 89: 252-257.

6. McNamara DM, Holubkov R, Starling RC, Dec GW, Loh E, et al. (2001) Controlled trial of intravenous immune globulin in recent-onset dilated cardiomyopathy. Circulation 103: 2254-2259.

7. Haque A, Bhatti S, Siddiqui FJ (2009) Intravenous immune globulin for severe acute myocarditis in children. Indian Pediatr 46: 810-811.

8. Strachan DP (1989) Hay fever, hygiene, and household size. BMJ 299: 12591260.

9. Okada H, Kuhn C, Feillet H, Bach JF (2010) The 'hygiene hypothesis' for autoimmune and allergic diseases: an update. Clin Exp Immunol 160: 1-9. 
Citation: El-Saiedi SA (2013) Randomized Controlled Trial on the Use of Intravenous Immune Globulin in Acute Pediatric Myocarditis. J Clin Res Bioeth 5: 170. doi:10.4172/2155-9627.1000170

Page 5 of 5

10. Weller AH, Hall M, Huber SA (1992) Polyclonal immunoglobulin therapy protects against cardiac damage in experimental coxsackievirus-induced myocarditis. Eur Heart J 13: 115-119.

11. Dwyer JM (1992) Manipulating the immune system with immune globulin. N Engl J Med 326: 107-116.

12. Finkel MS, Oddis CV, Jacob TD, Watkins SC, Hattler BG, et al. (1992) Negative inotropic effects of cytokines on the heart mediated by nitric oxide. Science 257: 387-389.

13. Hosenpud JD, Campbell SM, Mendelson DJ (1989) Interleukin-1-induced myocardial depression in an isolated beating heart preparation. $\mathrm{J}$ Heart Transplant 8: 460-464.

14. Arend WP, Smith MF Jr, Janson RW, Joslin FG (1991) IL-1 receptor antagonist and IL-1 beta production in human monocytes are regulated differently. J Immunol 147: 1530-1536.

15. Felix SP, Staudt A, Dorffel V (2000) Haemodynamic effect of immune adsorption and subsequent immunoglobulin substitution in dilated cardiomyopathy Three months results from randomized study. J Am Coll Cardiol 35: 1590-1598.

16. O'Connell JB, Robinson JA, Henkin RE, Gunnar RM (1981) Immunosuppressive therapy in patients with congestive cardiomyopathy and myocardial uptake of gallium-67. Circulation 64: 780-786

17. Anderson JL, Fowles RE, Unverferth DV, Manson W (1987) Immunosuppressive therapy of myocardial inflammatory disease: initial experience and future trials to de-fine indications for therapy. Eur. Heart J 8: 263-266

18. Parrillo JE, Cunnion RE, Epstein SE, Parker MM, Suffredini AF, et al. (1989) A prospective, randomized, controlled trial of prednisone for dilated cardiomyopathy. N Engl J Med 321: 1061-1068.

19. Tomioka N, Kishimoto C, Matsumori A, Kawai C (1986) Effects of prednisolone on acute viral myocarditis in mice. J Am Coll Cardiol 7: 868-872.
20. Al-Saeed S, Dilawar M (2008) Combined use of intravenous immuneglobulin and steroid for acute myo carditis in pediatric population. Heart Views 9: 137141

21. Giesecke $\mathrm{J}$ (1987) The long-term prognosis in acute myocarditis. Eur Heart $\mathrm{J}$ 8: 251-253.

22. Dec GW Jr, Palacios IF, Fallon JT, Aretz HT, Mills J, et al. (1985) Active myocarditis in the spectrum of acute dilated cardiomyopathies. Clinical features, histologic correlates, and clinical outcome. N Engl J Med 312: 885-890.

23. Olsen EGJ, Meany BT, Richardson PJ (1988) The role of biopsy in the diagnosis and follow up of myocarditis: a critical review. Schulthesiss, (ed.) New concepts in viral heart disease. Springer Verlag, Berlin, USA, 285-294.

24. Sinagra G, Maras P, D’Ambrosio A, Gregori D, Bussani R, et al. (1997) [Clinical polymorphic presentation and natural history of active myocarditis: experience in 60 cases]. G Ital Cardiol 27: 758-774.

25. D'Ambrosio A, Patti G, Manzoli A, Sinagra G, Di Lenarda A, et al. (2001) The fate of acute myocarditis between spontaneous improvement and evolution to dilated cardiomyopathy: a review. Heart 85: 499-504.

26. (2010) Myocarditis: Current Treatment Overview. Journal of Cardiac Failure 16

27. McNamara DM, Rosenblum WD, Janosko KM, Trost MK, Villaneuva FS, et al. (1997) Intravenous immune globulin in the therapy of myocarditis and acute cardiomyopathy. Circulation 95: 2476-2478.

28. Karjalainen J, Viitasalo M, Kala R, Heikkilä J (1984)24-Hour electrocardiographic recordings in mild acute infectious myocarditis. Ann Clin Res 16: 34-39.

29. Karjalainen J (1983) Functional and myocarditis-induced T-wave abnormalities Effect of orthostasis, beta-blockade, and epinephrine. Chest 83: 868-874.

30. Kindermann I, Barth C, Mahfoud F, Ukena C, Lenski M, et al. (2012) Update on myocarditis. J Am Coll Cardiol 59: 779-792. 